\title{
Paroxysmal atrioventricular block precipitated by an atrial premature beat. What is the mechanism?
}

\author{
S. Serge Barold ${ }^{1}$, Roland X. Stroobandt ${ }^{2}$ \\ ${ }^{1}$ Florida Heart Rhythm Institute, Tampa, Florida, USA \\ ${ }^{2}$ Heart Center, Department of Electrophysiology, University Hospital, Ghent, Belgium
}

\section{Introduction}

A 65 year-old woman presented with two episodes of syncope. She developed paroxysmal atrioventricular (AV) block when an ECG was being recorded (Fig. 1). She developed at least one other similar episode. Then, she developed complete AV block with a stable escape ventricular rhythm of about $30 \mathrm{bpm}$ during which an atrial premature beat was conducted (Fig. 2). Isoproterenol was administered which increased the ventricular rate to $47 \mathrm{bpm}$. The patient remained stable and later received a permanent dual chamber pacemaker.

Lee et al. [1] reported 68 cases of bradycardiadependent AV block (30 cases [group I] were from the investigators' two institutions and 38 cases were added from the literature [group II]) [2-5]. Paroxysmal AV block (PAVB) was defined as the abrupt occurrence of pause-dependent (phase 4) AV block in a patient with apparently normal 1:1 AV conduction between the episodes of PAVB [1]. Most patients present with syncope or near-syncope. There were 19 of 68 patients $(28 \%)$ with a normal ECG as in our patient (Fig. 1). Thus, a normal ECG does not rule out PAVB. This form of AV block can be precipitated by atrial or ventricular premature beats, His bundle extrasystoles, sinus slowing either spontaneous or induced by carotid sinus massage and rarely after supraventricular tachycardia by overdrive suppression upon termination. In

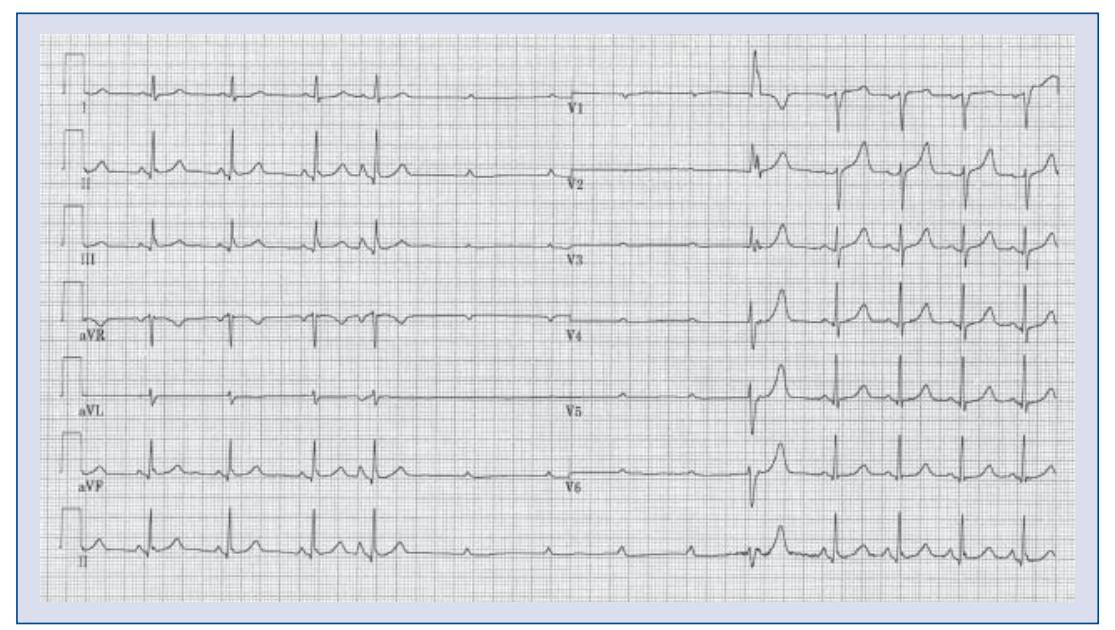

Figure 1. On the left a 12-lead ECG shows sinus rhythm at a rate of about $73 \mathrm{bpm}$. An atrial premature beat precipitates paroxysmal atrioventricular block (PAVB) with prolonged ventricular asystole during which the sinus rate increases to $83 \mathrm{bpm}$. A ventricular escape terminates the PAVB. During 1:1 AV conduction, the ECG is normal with a narrow QRS complex. See text for details.

Address for correspondence: S. Serge Barold, MD, Florida Heart Rhythm Institute, Tampa, Florida, USA, tel: 8138911922 , e-mail: ssbarold@aol.com 


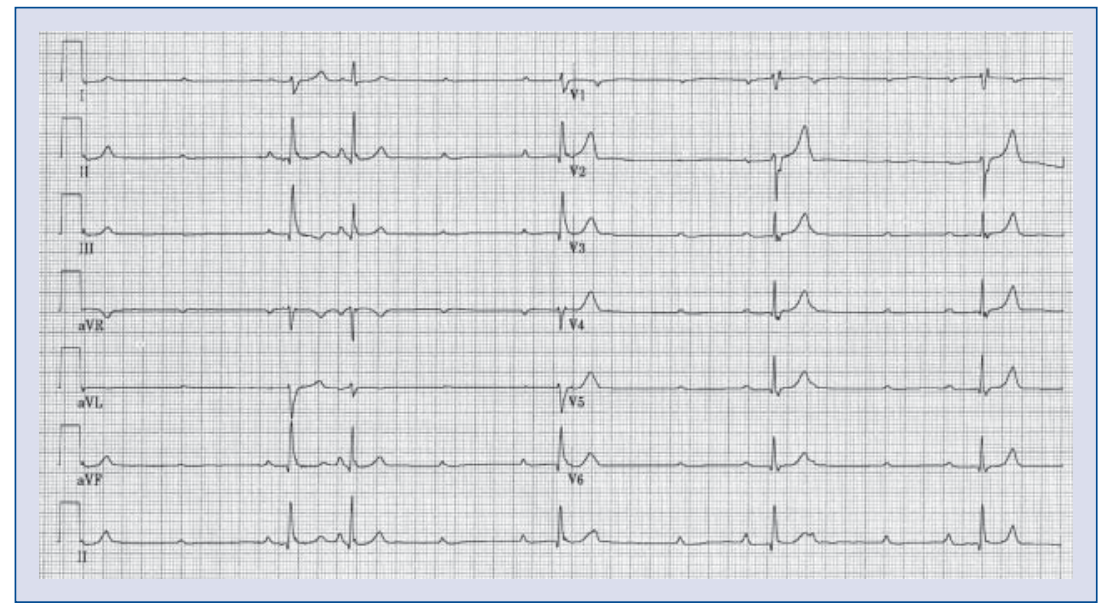

Figure 2. 12-lead ECG showing complete atrioventricular block with a stable ventricular escape rhythm close to $30 \mathrm{bpm}$. An atrial premature beat is conducted to the ventricles. See text for details.

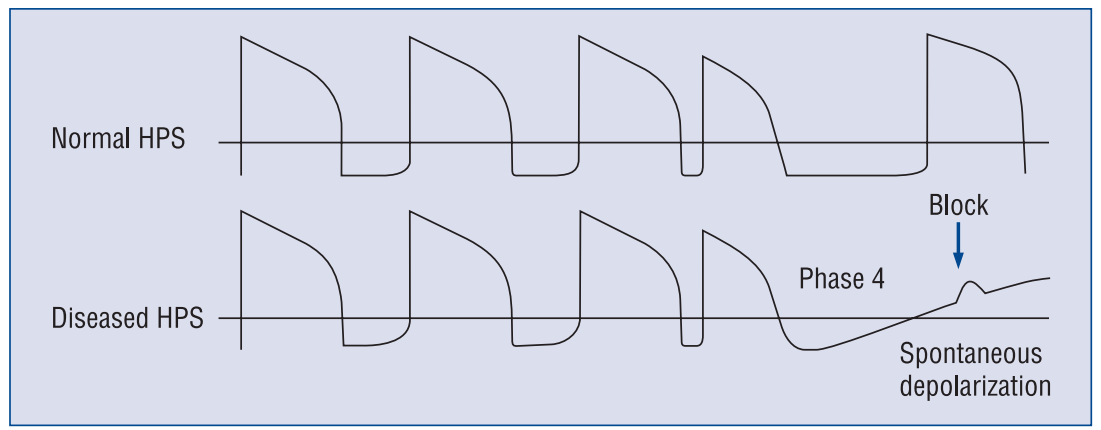

Figure 3. Diagramatic representation of phase 4 block in the His-Purkinje system (HPS). Legend: Action potentials in the normal and diseased conduction system showing phase 4 block. Adapted from reference [1] with permission.

group I, an atrial premature beat precipitated PAVB in 9 of 30 patients (30\%) in the same way as in our patient [1]. Typically the relatively long runs of ventricular asystole are terminated with resumption of AV conduction by an appropriately timed by a somewhat unreliable ventricular escape or premature beat which invades the site of block retrogradely and resets the transmembrane potential. Less commonly an increase in the sinus rate permits return of 1:1 AV conduction.

\section{Mechanism}

PVAD as defined is always due to disease in the His-Purkinje system. Abnormal His-Purkinje cells may acquire the property of spontaneous phase 4 depolarization when the sodium channels are inactive. During a long pause, the site of the diseased His-Purkinje abnormality continues to depolarize and become less responsive to subsequent impulses which are then blocked (Fig. 3). AV conduction usually resumes with an appropriately timed escape or premature beat that resets the membrane potential to its resting state.

\section{Diagnosis}

Because PAVB is typically precipitated by slowing of the heart rate, it may be misinterpreted as a vagally mediated mechanism [1]. This differential diagnosis is important because PAVB is a clear indication for permanent pacing while vagally mediated block is not. Lee et al. [1] provided an elegant way to differentiate the two entities. Briefly, a vagal mechanism produces gradual changes (rather than abrupt ones with PAVB) and causes simultaneous sinus slowing and AV block (with a long PR interval or a type I second-degree equivalent), and 
there is slowing of the sinus rate during the period of AV block. In contrast in PAVB the sinus rate may accelerate during ventricular asystole but it does not affect the AV block. The history is important because situations known to cause a vagal reaction may suggest a vagal mechanism. The role of an electrophysiologic study (EPS) in PAVB remains uncertain. EPS is specific but not sensitive. A normal HV interval and a negative EPS do not rule out the diagnosis of PAVB.

\section{Relationship of paroxysmal AV block to stable complete AV block}

Bortone et al. [2] described the occurrence of bradycardia dependent PAVB in a patient with an acute inferior wall myocardial infarction complicated by complete AV nodal block and an accelerated AV junction rhythm (70-75 bpm). A ventricular premature complex precipitated PAVB with a long period of ventricular asystole. Such an observation suggests that PAVB occurred on the basis of a phase 4 block but the underlying stable complete AV block was not due to phase 4 block. In our patient a stable escape ventricular rhythm became established after the initial manifestations of PAVB. The development of such a stable ventricular escape rhythm is rare in patients presenting with PAVB where escape rhythms are highly unpredictable. Figure 2 shows how a premature atrial beat was normally conducted to the ventricles in the setting of complete AV block with a stable escape ventricular rhythm. This response is virtually the exact opposite of the observations of Bortone et al. [2]. This suggests that complete AV block (with a stable ventricular rhythm during) was itself sustained by a phase 4 mechanism and the atrial premature beat was conducted because the shorter interventricular interval did not meet the critical cycle prolongation to produce phase 4 block. In the past, conduction of the atrial premature beat would have been attributed to supernormal conduction.

\section{Phase 3 and phase 4 blocks}

Phase 4 block may sometimes occur in combination with tachycardia dependent (phase 3 ) block. In this situation there is a relatively narrow range of rates between the critical heart rates for the development phase 3 and phase 4 blocks where normal conduction can occur $[5,6]$.

Conflict of interest: none declared

\section{References}

1. Lee S, Wellens HJ, Josephson ME. Paroxysmal atrioventricular block. Heart Rhythm, 2009; 6: 1229-1234.

2. Bortone A, Albenque JP, Marijon E, Donzeau JP. Complete atrioventricular block and asystole in a patient with an inferior acute myocardial infarction: What is the mechanism? Heart Rhythm, 2008; 5: 1077-1079.

3. Motté G, Desoutter P, Olive B, Bodereau P, Welti JJ. Paroxysmal block in phase 4 of the bundle of His. Arch Mal Coeur Vaiss, 1977; 70: 797-807.

4. Coumel P, Fabiato A, Waynberger M, Motte G, Slama R, Bouvrain Y. Bradycardia-dependent atrio-ventricular block. Report of two cases of A-V block elicited by premature beats. J Electrocardiol, 1971; 4: 168-177.

5. Rosenbaum MB, Elizari MV, Levi RJ, Nau GJ. Paroxysmal atrioventricular block related to hypopolarization and spontaneous diastolic depolarization. Chest, 1973; 63: 678-688.

6. Barold SS, Ong LS, Young JA. Electrocardiographic observations in bradycardia and tachycardia-dependent atrioventricular block. Relationship to supernormal phase of intraventricular conduction. Chest, 1975; 67: 450-457. 